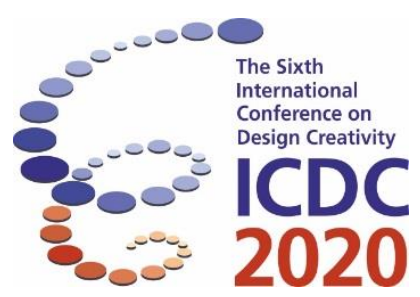

\title{
Fixation in the Creative Practices and Perceptions of Independent Ceramic Designers
}

\author{
Qianang Sun, Eunyoung Kim \\ School of Knowledge Science, Japan Advanced Institute of Science and Technology, Nomi, \\ Japan
}

\begin{abstract}
Fixation, as a mental activity, is usually discussed in the design process and creative expression. Several studies have conducted experiments aiming to find how to reduce the fixation in design processes and enhance creative expression. However, there is little concern about how designers deal with the fixation in their real works so as to improve the understanding of design fixation effect on the design process. Ceramic design, as a practice-based zone of design, has contributed to people's daily lives not only as a utilitarian product, but also as a means to meet increasingly unique and aesthetic needs through creative outcomes. In this study, we propose to explore the designer's perceptions of fixation, which is embedded in their practice process through interviewed independent ceramic designers. Thirteen participants have been recruited, and we have adopted a thematic analysis in this research. The findings show that three elements should be noted in addressing the fixation involving 1) Repetition, 2) Transformation and 3) combination. Furthermore, the design fixation should be discussed with critical thinking by combining case studies in the future. This study will benefit craft designers and related stakeholders, and also provide a reference for design education.
\end{abstract}

Keywords: Design, Fixation, Ceramic Design, Design process, Creative expression

\section{Introduction}

Arnheim (1965) argues that creativity should be defined as the full development of individual awareness, action and perception. With this aspect, exploring the practice process in a creative experience becomes an essential way of investigating creativity. Despite incomputable mental activities happening in the creative process, the term "fixation", the barriers to insight are often caused by the adverse effects of prior knowledge (Jansson \& Smith, 1991). This phenomenon and its variants have been studied in several classic experiments like Maier (1931) and Duncker and Lee (1945) who have demonstrated how people's obsession with the traditional functions of artefacts inhibited their ability to discover new services. It is observed that, when craft designers are creating the products in the real world, they performed naturally to replay a skilful action to build a useful ware with prior experience or patterns, which also inhibited their ability to develop a new feature. However, the subtle perception of design fixation in practice-based design often has been overlooked as well as arbitrarily treated as a negative factor, which should reduce it. For example, Jansson and Smith (1991) summarised the design fixation as "a blind, sometimes counterproductive, adherence to a limited set of ideas in the design process", moreover, it has applied to many areas of design practice, including industrial design, architecture, engineering, and software design, with various measures proposed for avoiding and reducing it (Odette 
da Silva, Nathan Crilly, \& Paul Hekkert, 2015). In this study, we focus on ceramic design where a typical practice-based design stands at a crossroad of skilful activity and artful expression by coherence perceptions in the creative process.

As a traditional view, ceramic creation presented a sophisticated knowledge of body and practices (Negus \& Pickering, 2004), which most times is the antithesis of creativity (Wagoner, 2017). However, it is the visible change from traditional handmade switch to industrial production in ceramic production, particularly along with modern design education and advanced technology. Several ceramic designers established an independent studio to produce stylised products, which are fundamentally different from obsolete production. Therefore, we conduct the research proposed to explore the creative experiences of independent designers to investigate their practice process and the perceptions of design fixation. We have carried out semi-structured interviews with thirteen ceramic designers, and thematic analysis has adopted for data analysis.

In the following sections, the article is organised is four parts. First, we searched the previous research of fixation, which examines design work from different aspects. Then, we will describe the research method employed in this paper, illustrate the information of data collection, and analyse the data with thematic analysis. Finally, the findings will be shown in the result, and a discussion then follows. The main contribution of this study is to provide a new insight to enrich the understanding of design fixation addressed in ceramic design and, furthermore, to provide a reference for craft design research.

\section{Literature Review}

Many studies of design fixation indicated that if designers have been exposed to an existing design solution (e.g., from their current environment), this may unwittingly narrow the scope of a creative idea they are exploring (e.g., by repeating the characteristics of existing solutions). Jansson and Smith's (1991) experiment reveals that designers often replay the critical features from the prior solution they have seen and performed advertently, even impact on how did they explain the problems.

Furthermore, this behaviour persists even when the designers received instructions to avoid repeating specific features of these examples. The recent research from Junaidy, Nagai, \& Ihsan, (2013a) has explored in-depth cognitive levels at the early stage of idea generation in a comparative analysis of artisans. They found the artisans tend to activate low-weighted associative concepts with a smaller number of polysemous features (multiple meanings) compared to modern designers (Junaidy, Nagai, \& Ihsan, 2013b). This finding was also taken to explain their concern for substantive issues such as proportion and shape; meanwhile, designers brought up other contextual elements, even referring to user preferences (Wagoner, 2017). With these results, we can realise that the cognitive level represents different ways of thinking in the design process, and the abstract transformation is a prerequisite for avoiding negative effects of design fixation (Goldschmidt, 2011). However, the occurrence of abstraction and change mostly depends on the essential of stimulus and personal attributes.

In contrast, there are many advantages to using existing features as a reference for generating new ideas during the constructive stage. It might reduce the time and receive acceptance quickly (if the previous solution was conventional), and even enhance the designers' confidence. Particularly in practice-based design, reuse of the traditional patterns is commonly acceptable because the knowledge of how to shape the product is embedded in previous examples (Jacobs-Lawson \& Hershey, 2005). Moreover, re-shaping the classic patterns or figures is the shortcut to understanding the practical technique, which cannot communicate with language. Even though this kind of perceived process may have an impact on the following actions or prevent the innovate ideas (this may be bad), if designers are encouraged to explore the fixation to narrow feasible solutions, which may save time (Luchins, 1946), thereby reducing the cost of a design project, this may be useful. Nevertheless, we know less about designers' perceptions when they suffered a dilemma in the real process and how do they overcome the fixation. Relying on the prior studies, we would like to explore the professional operation of ceramic design by interviewing early-career designers to identify the factors discouraging design fixation in the independent creation. It is meaningful for theory development and empirical research in the next stage. 


\section{Methods}

Although design fixation is an applied aspect of human behaviour, the choice of research method is restrictive and usually examined in the experimental set by using statistical approaches. Even though there are a few related qualitative studies, they are still isolated from the core empirical literature (Neroni \& Crilly, 2019). Furthermore, verbalising one's thoughts in the highly embodied practice's process are complicated and potentially distracting for the participants (Yokochi \& Okada, 2010). It is also hard to use methods like think-aloud protocols (Junaidy \& Nagai, 2013) to capture implicit thinking. Therefore, we have conducted a qualitative approach by using semi-structured interviews with thirteen ceramic designers to explore their perceptions of the fixation in the practice process. The reason we choose independent designers as participants in this study was due to the long-term observation. At first, the increasing numbers of designers established the personal studio to produce original products. However, the modern ceramic designers (in fact, almost all discipline designers) usually design their products in an independent space, even they are in the teamwork or employed in a company. The semi-structured interview was conducted in one-to-one conversations with thirteen ceramic designers in the past two months to collect the data. It commonly takes two hours, and 3 of the participants took twice as long during the collection. Thematic analysis has been adopted in data analysis to reveal the subtle perceptions in ceramic design.

\subsection{Data Collection}

In this study, we recruited thirteen participants who are engaged in making different ceramic wares (See Table1). Their creative experiences could accumulate extensive knowledge and the potential to provide an appropriate description of the feeling and thinking. The distinguished perception is embedded in different education background enhances the quality of the interviews. Four females and six males participated in the discussion individually online, while three interviews took place at their workplace and a design course. In the next phase, we will analysis the interview contents to explain the findings of fixation in ceramic design and how do the designers overcome it.

Table 1. Basic information of participants

\begin{tabular}{lccc}
\hline Interviewee & Age & Educational Background & Typical Work \\
\hline $1 \mathrm{~T}$ & 32 & Installation Art & Teapot \\
$2 \mathrm{~W}$ & 32 & Ceramic Art & Tableware \\
$3 \mathrm{~S}$ & 29 & Ceramic Art & Tableware \\
$4 \mathrm{~W}$ & 31 & Ceramic Art & Tableware \\
$5 \mathrm{D}$ & 30 & Painting & Vase \\
$6 \mathrm{~T}$ & 30 & Ceramic Art & Tableware \\
$7 \mathrm{D}$ & 29 & Sculpture & Teapot \\
$8 \mathrm{H}$ & 33 & Glass \& Ceramic & Lamp \\
$9 \mathrm{~J}$ & 35 & Ceramic Art & Vase \\
$10 \mathrm{M}$ & 31 & Painting & Vase \\
$11 \mathrm{~B}$ & 32 & Sculpture & Tableware \\
$12 \mathrm{G}$ & 31 & Ceramic Art & Teapot \\
$13 \mathrm{X}$ & 30 & Visual Design & Vase \\
\hline
\end{tabular}

\subsection{Data Analysis}

We transcribed the recordings of the related conversations and augmented this with several handwritten notes during the interviews. The transcripts involved 4,960 words and analysis through reading, sorting, 
re-reading and coding to explore the possible meanings as much as possible. We referenced the innovation process model (Meinel \& Leifer, 2011) that has been summarised in three active functions called "Plan", "Execute", and "Synthesis". This model provides a space to discuss the emerged themes in this study, which are "Repetition", "Transformation", and "Combination" (See Figure1). The three themes have been sorted from the interview with participants' description. The number of utterances in each category is 1036 words with the theme "repetition", 796 words with "transformation" and 838 words with "combination". Subsequently, we discussed the emerged themes using the design process embedded in the Unified Innovation Process Model (Meinel \& Leifer, 2011)

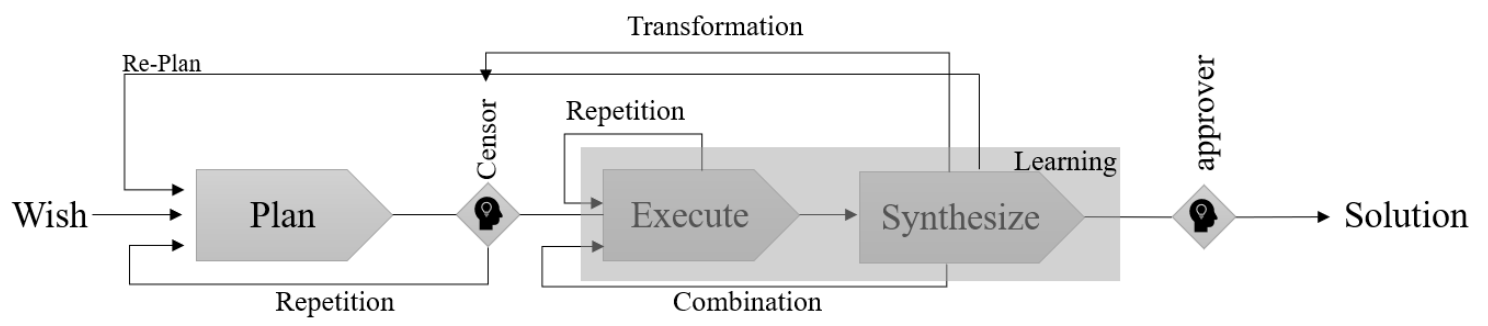

Figure. 1 Unified Innovation Process Model (Meinel \& Leifer, 2011)

\subsubsection{Repetition}

The excellent design lies in the full development of divergent thinking, the preparation of alternatives, and a great deal of empirical knowledge and specific evaluation criteria. For a practice-based design, experiential knowledge mainly comes from the skill training. The traditional artisans were skilful but submerged their style in the monotonous work, and the modern designers are still not exempt from the tedious, repetitive process at the primary stage. However, the participants stressed that in this phase that repetitive work promotes critical thinking in their creative process (See Table2).

It was found that designers tended to revise the details in the continuous repetition process to improve the project or awaken new ideas to avoid the design fixation. In the craft design, the actual operation is an essential cognitive process. At the conception stage, designers expect to generate inspiration or follow the intuition based on external stimuli, such as several examples, picture associations and sketches. However, these initial ideas usually failed in practice due to the material limitation or technique mistakes. The repetition phase provides an opportunity to replay the practice process to explore the problem space by concentration.

\subsubsection{Transformation}

Transforming the reference resource is an essential ability for designers across the domain and it is a critical step to visualise the fragmentary stimuli and constructs a creative outcome. This phase consequently emerged at the conceptual level. Moreover, a promising design is not only focused on the function but also designed to enrich users' experience mainly in daily life design like ceramics. In exploring the transcript, we have found that transformation performed as a meaningful process extended the expression space to address fixation and screen out the best solution in ceramic design (See Table $3)$.

The design fixation generally is described as the fundamental tension between two opposing characteristics. With this background, the conceptual development demands of the designers should be keeping an open mind to embrace more possibilities and insisting on an idea, which is worth to develop. However, when the problem comes to the practice part, the solution depends on the individual psychological state. Furthermore, a broad range of personal, organisational or contextual factors influences the degree that design fixation occurs and the effect on creative outcomes.

\subsubsection{Combination}

"Combination" seems an overused word in the design domain. In this study, it was mentioned by participants to mean a kind of exploration in the overall process when they felt stressed or tended to 
examine a new concept. Experimenting with diverse materials or techniques is an efficacy way to get new knowledge or find new solutions in ceramic design. In other words, rarely do designers always concentrate on the original material or skill to conduct their design (See Table4).

Table2. Interview contents extract showing the example of "Repetition."

\begin{tabular}{|c|c|}
\hline Interviewee & Description \\
\hline Interviewee $13 X$ & $\begin{array}{l}\text { "Sometimes, I feel that my hands are talented than me. When I } \\
\text { am working on the wheel, I can feel my hands leading my } \\
\text { brain. The line flowed and perceived by the hand. Repeating an } \\
\text { action or a program will allow me to temporarily follow my } \\
\text { intuition to feel the power of the clay. This state may last for a } \\
\text { long time, but the advanced understanding also has brewed in } \\
\text { this period." }\end{array}$ \\
\hline Interviewee $1 T$ & $\begin{array}{l}\text { "Inspiration is very unreliable. My intuition comes from } \\
\text { much meaningless practice. Because the creation, } \\
\text { especially craft art, never completed efficiently. When I } \\
\text { was in trouble, I also attempt to choose others' solutions } \\
\text { or take example by classic case while it was easy to } \\
\text { succeed. However, I am afraid to fix other people's } \\
\text { creation while losing my style." }\end{array}$ \\
\hline Interviewee $2 \mathrm{~W}$ & $\begin{array}{l}\text { "I have received much tacit knowledge of ceramic } \\
\text { making by re-built the classic shape, mainly when I lose } \\
\text { the direction; I will go back to repeat the previous work } \\
\text { attempt to warm up. On the hand, I can relax in a } \\
\text { conversant program and on the other hand, I am looking } \\
\text { forward to getting new ideas to revise the details of } \\
\text { recent work." }\end{array}$ \\
\hline Interviewee $9 \mathrm{~J}$ & $\begin{array}{l}\text { "I think the ceramic design is a process fulfilling by skills. } \\
\text { The first thing is to understand what do you going to do and } \\
\text { how to do it. Copy the successful work and repeat it } \\
\text { thousands of times seems tedious, but you can not create a } \\
\text { talent product without this training." }\end{array}$ \\
\hline
\end{tabular}

Table3. Interview contents extract showing the example of "Repetition."

\begin{tabular}{l} 
Description \\
\hline Interviewee \\
\hline Interviewee $10 M \quad$ Most of my works were start with the depiction of nature- \\
the scenery in the morning, the colour of autumn or the \\
texture of stones. I would like to combine different elements \\
to transform my feeling and describe them on my vessel. It \\
is not as simple as to paste a leaf or takes a photo. Because \\
of the fire will give me a hand at the final step until I finish \\
the magic process." \\
"The practice process means disassembly and \\
reorganization. You'd better to subtract any random \\
patterns to make an object stand the test of time. The \\
usage is another way to create it by your behaviors. So I \\
will transform the roles to evaluate my works. Then I \\
will get more critical thinking from different aspects to \\
correct them." \\
"It is never easy if you want to make a pot deeply \\
touching users' mind even lead them a new life. That \\
sounds exceptionally abstract because you do not know \\
what the best products look like, and the only things you \\
can do is to explore the resources that called an \\
inspiration to conceive, overthrow, and re-build, \\
infinitely close to the best solution."
\end{tabular}


Table4. Interview contents extract showing the example of "Combination."

\begin{tabular}{|c|c|}
\hline Interviewee & Description \\
\hline Interviewee $3 S$ & $\begin{array}{l}\text { "I am interested in collecting the clay from the natural and } \\
\text { categorize in different colors and qualities. I try different } \\
\text { combinations when I have no ideas about my work, and } \\
\text { these various materials and tools usually pull me out of the } \\
\text { problematic condition which may produce a very novel } \\
\text { color or texture." }\end{array}$ \\
\hline Interviewee $5 D$ & $\begin{array}{l}\text { "I keep exploring different materials, tools and patterns } \\
\text { to prevent indulging in a single solution with the same } \\
\text { skill even fixed idea. Personally, the ceramic design is a } \\
\text { co-creation process of human, clay, water and fire. } \\
\text { Although human is the subject in the process, there are } \\
\text { still much uncertainty seems to cause failure but make } \\
\text { the creative process attractively." }\end{array}$ \\
\hline Interviewee $12 G$ & $\begin{array}{l}\text { "The extended period production indicate the ceramic } \\
\text { design need more carefully in each stage. I hope my } \\
\text { work is actively not only to complete the customized } \\
\text { products. It is necessary to request new creations instead } \\
\text { of following or copying previous works even is my } \\
\text { successful design. That may be extremely challengeable } \\
\text { for a designer." }\end{array}$ \\
\hline
\end{tabular}

Design is a process of problem-solving widely accepted so far. The term design fixation often is treated as a kind of unconscious or habitual behaviour might harm creative expression. However, from the interview content, we found that the participants usually have the conscious of their limitations in the creative process - the interaction of designers and their works throughout the materials, skills, and ambiguous problem spaces. When a primary step has been completed, and the next plot is not clear, or they feel fixed in a passive condition; they can detect that and try to find a way to break out. With combination, whether it is the combination of material or technology, they start to find solutions before the fixation happening. It could be an effective way for ceramic designers to prevent fixation so as to stay creative and develop new ideas.

\section{Results}

The themes discussed above, repetition, transformation, and combination are the efficient elements to develop a better design by addressing the fixation in ceramic design, especially the ceramic creation, represent a high-contents practical topic. As such, these three themes cannot be isolated in each phase of creation; in contrast, they interact and even playback during the practice process of design. At the micro-level, the three activity functions repetition, transformation and combination are waved in the design process. Moving to the macro view, both transformation and combination are associated with repetition. This interaction could generate a path to reach the pattern or plot and approach to raise new creative experiences. In this article, the designers we interviewed expressed a rich understanding and experience of ceramic design. They have shown the flexibility, sensitivity, and passion for creation that enable them to face the obstacles in the creative process. They are more relaxed and calm in the actual creation, and such performances are entirely different from the laboratory experiment.

As a practice-based design, designers need to understand the object and users' expectations. Through the data collection and analysis, keep a balance between concept and materials is an implicit target in the creative process. Because our interviewees have considerable professional experience, we gained indepth reflective perceptions about design fixation from the interviews. Moreover, when we reviewed the transcripts, it can be realised that "time" is a crucial factor that should be considered. The production 
cycle allows designers to conceive, implement, test, reflect, and practice over time. Experience accumulation in a period may cause a subtle change in their attitude for design fixation. Therefore, we believe that the adverse effects can be prevented by appropriate reflection even though the design fixation is a problematic issue to gain control of it (Odette da Silva et al., 2015).

It is worth noting that removing the design fixation in ceramic design is not always a bad thing. On the contrary, the participants perceived the tension of opposites but still fulfilled the time by repetition, particularly in the modelling phase. They believe that quantitative change leads to a qualitative shift in this process. This phase is different from blindly following a fixed rule or other cases, and sometimes helps clarify the problem space. However, even the process to clarify the problem space seems to spontaneously extract the task that designers need to solve in the real environment, but such a primary stage also required insight-based ability to manipulate the whole practice.

Even though there are a number of considerable studies on design fixation, however, the mental activities are individual in each field. More extensively, the fixation may hinder the fluency and originality of creative expression, but on the other hand, it can help designers accumulate experience in practice from the reflective viewpoint. Through the interviews and thematic analysis, this study concludes that three factors played crucial roles in the creative process of ceramic making, including transformation, repetition, and combination, which impact the designers' perceptions of design fixation. Meanwhile, it provides a panoramic lens to reflect on designers' perceptions when they suffered a severe period constrained by fixed ideas in their creative process of ceramic making.

\section{Discussion}

Design fixation is a mental state that may hinder creative expression. However, fixation and creativity should be considered from a broader perspective. The Russian scholar Sekhorovsky proposed that the artistic design is a de-familiarising process, which aims to increase the difficulty and prolong the time in perception by making difficulties in forms. Therefore, keeping critical thinking on design fixation is more valuable compared to following it blindly. Moreover, creativity also needs to be developed following an appropriate evaluation. How to balance these two forces while focusing on problemsolving is worth future study.

Additionally, in modern design education, heuristic strategies are widely used. However, in actual design works, which involve the required problem space, assessment phase and decision-making, when a designer relies on the thinking skill that cultivated in the undergraduate stage to search for the shortcuts to reduce the complexity of judgment, thus is unable to get an objective assessment, it is likely to cause fixation and lead to incorrect results. On this point, case studies should be adopted to gain a deeper understanding of the fixation effect on creativity across different levels.

The limitations of this research lie in the fact that it is only focused on the individual perception of fixation in the creative process without any other approaches to support the result. Further research should carry out case studies and group discussions to generate quantitative data to explore the hidden influences of design fixations and to provide references for design education in the future.

Finally, we would like to end the paper with one reflection from ceramic making, which is that the design in any field is a continuous process with several stagnant stages seems meaningless. Nevertheless, the change indicated in our findings is one of the ways to achieve successful work. The recipe is to confront the fixation and keep creating.

\section{References}

Arnheim, R. (1965). Art and visual perception: A psychology of the creative eye. Univ of California Press.

Duncker, K., \& Lees, L. S. (1945). On problem-solving. Psychological Monographs, 58(5), i.

Goldschmidt, G. (2011). Avoiding design fixation: Transformation and abstraction in mapping from source to target. Journal of Creative Behavior, 45(2), 92-100. https://doi.org/10.1002/j.2162-6057.2011.tb01088.x

Jacobs-Lawson, J., \& Hershey, D. (2005). Influence of Future Time Perspective, Financial Knowledge, and Financial Risk Tolerance on Retirement Saving Behaviors. Financial Services Review, 14(4), 331.

Jansson, D. G., \& Smith, S. M. (1991). Design fixation. Design Studies, Vol. 12, pp. 3-11. https://doi.org/10.1016/0142-694X(91)90003-F 
Junaidy, D. W., \& Nagai, Y. (2013). The in-depth cognitive levels of imagination of artisans and designers. J. of Design Research, 11(4), 317. https://doi.org/10.1504/jdr.2013.057761

Junaidy, D. W., Nagai, Y., \& Ihsan, M. (2013a). Craftsmen versus designers: the difference of in-depth cognitive levels at the early stage of idea generation. In ICoRD'13 (pp. 223-234). Springer.

Junaidy, D. W., Nagai, Y., \& Ihsan, M. (2013b). Craftsmen Versus Designers: The Difference of In-Depth Cognitive Levels at the Early Stage of Idea Generation. 223-234. https://doi.org/10.1007/978-81-322-1050-4_18 Luchins, A. S. (1946). Classroom experiments on mental set. The American Journal of Psychology, 59(2), 295298.

Maier, N. R. F. (1931). Reasoning in humans. II. The solution of a problem and its appearance in consciousness. Journal of Comparative Psychology, 12(2), 181.

Meinel, C., \& Leifer, L. (2011). [Articles inside] Understanding Innovation Series Editors - Hasso Plattner. Retrieved from http://www.springer.com/series/8802

Negus, K., \& Pickering, M. J. (2004). Creativity, communication and cultural value. Sage.

Neroni, M. A., \& Crilly, N. (2019). Whose ideas are most fixating, your own or other people's? The effect of idea agency on subsequent design behaviour. Design Studies, 60, 180-212. https://doi.org/10.1016/j.destud.2018.05.004

Odette da Silva, Nathan Crilly, \& Paul Hekkert. (2015). How People's Appreciation of Products Is Affected by Their Knowledge of the Designers' Intentions. International Journal of Design, 9(2), 21-33. Retrieved from http://ijdesign.org/index.php/IJDesign/article/view/1961/692

Wagoner, B. (2017). The Palgrave Handbook of Creativity and Culture Research. In The Palgrave Handbook of Creativity and Culture Research. https://doi.org/10.1057/978-1-137-46344-9

Yokochi, S., \& Okada, T. (2010). Creative Cognitive Process of Art Making: A Field Study of a Traditional Chinese Ink Painter Creative Cognitive Process of Art Making : A Field Study of a Traditional Chinese Ink Painter Sawako Yokochi and Takeshi Okada. Creativity Research Journal, (932223628). https://doi.org/10.1207/s15326934crj1702 